\title{
ORBITAL STABILITY OF STANDING WAVES FOR A CLASS OF SCHRÖDINGER EQUATIONS WITH UNBOUNDED POTENTIAL
}

\author{
GUANGGAN CHEN, JIAN ZHANG, AND YUNYUN WEI
}

Received 26 August 2004; Revised 20 October 2004; Accepted 9 November 2004

This paper is concerned with the nonlinear Schrödinger equation with an unbounded potential $i \varphi_{t}=-\triangle \varphi+V(x) \varphi-\mu|\varphi|^{p-1} \varphi-\lambda|\varphi|^{q-1} \varphi, x \in \mathbb{R}^{N}, t \geq 0$, where $\mu>0, \lambda>0$, and $1<p<q<1+4 / N$. The potential $V(x)$ is bounded from below and satisfies $V(x) \rightarrow \infty$ as $|x| \rightarrow \infty$. From variational calculus and a compactness lemma, the existence of standing waves and their orbital stability are obtained.

Copyright (c) 2006 Hindawi Publishing Corporation. All rights reserved.

\section{Introduction}

In this paper, we consider the nonlinear Schrödinger equation with an unbounded potential

$$
i \varphi_{t}=-\triangle \varphi+V(x) \varphi-\mu|\varphi|^{p-1} \varphi-\lambda|\varphi|^{q-1} \varphi, \quad x \in \mathbb{R}^{N}, t \geq 0
$$

where $\mu>0, \lambda>0$, and $1<p<q<1+4 / N$. The potential $V(x)$ is bounded from below and satisfies $V(x) \rightarrow \infty$ as $|x| \rightarrow \infty$. Equation (1.1) has its physical background. For example, when $V(x)=|x|^{2}$, it models the Bose-Einstein condensate with attractive interparticle interactions under magnetic trap $[2,7,11,17,20]$.

When $\left|D^{\alpha} V\right|$ is bounded for all $|\alpha| \geq 2$, in terms of the smoothness of the time 0 of Schrödinger kernel for potentials of quadratic growth provided by Fujiwara [9], Oh [13] established the local well-posedness of (1.1) in the corresponding energy space. Since Yajima [19] showed that for superquadratic potentials, the Schrödinger kernel is nowhere $C^{1}$, we see that quadratic potentials are the highest-order potential for local well-posedness of (1.1). Thus the result of Oh [13], the local well-posedness of nonlinear Schrödinger equation with the potential function $V(x)$, is indeed sharp.

We are interested in the following standing waves of (1.1):

$$
\varphi(t, x)=e^{i w t} u(x)
$$

Hindawi Publishing Corporation Journal of Applied Mathematics and Stochastic Analysis Volume 2006, Article ID 57676, Pages 1-7

DOI 10.1155/JAMSA/2006/57676 
where $w \in R$ is a parameter and $u(x)$ is the solution of the nonlinear elliptic equation

$$
-\triangle u+V(x) u+w u-\mu|u|^{p-1} u-\lambda|u|^{q-1} u=0 .
$$

The interesting topics to investigate standing waves are pursued strongly by many physicians and mathematicians $[4,3,12,14,16]$.

For (1.3), Ding and Ni [8] by using "mountain pass" and comparison arguments got the existence of positive solutions. Rabinowitz [15] and Zhang [20,21] also studied the existence of the solutions for (1.3) by the method of variation. Hirose and Ohta [10] studied the uniqueness of the solution for (1.3).

In this paper, for $1<p<q<1+4 / N$, we establish the existence of the standing waves with the ground state of (1.1) by variational calculus which originates in Berestycki [1], Cazenave and Lions [6], Weinstein [18], and Zhang [20-23]. Furthermore, we prove the standing waves are orbitally stable.

This paper is organized as follows. In the second section, we give some necessary preliminaries which include the compactness lemma. In the third section, we prove the existence of the standing waves. And in the last section, we obtain their orbital stability.

\section{Preliminaries}

For (1.1), we impose the initial value as follows:

$$
\varphi(x, 0)=\varphi_{0}(x), \quad x \in \mathbb{R}^{N} .
$$

In the course of nature, we set

$$
H:=\left\{u \in H^{1}\left(\mathbb{R}^{N}\right): \int V(x)|u|^{2} d x<\infty\right\} .
$$

Here and hereafter, for simplicity, we denote $\int_{\mathbb{R}^{N}} d x$ by $\int d x$. H becomes a Hilbert space, continuously embedded in $H^{1}\left(\mathbb{R}^{N}\right)$, when endowed with the inner product

$$
\langle\varphi, \psi\rangle_{H}=\int \nabla \varphi \nabla \bar{\psi}+\varphi \bar{\psi}+(V(x)-\inf V(x)) \varphi \bar{\psi} d x,
$$

whose associated norm is denoted by $\|\cdot\|_{H}$.

Lemma $2.1[5,13]$. Let $V(x)$ satisfy that $\inf V(x)>-\infty$ and for each $|\alpha| \geq 2,\left|D^{\alpha} V\right|$ is bounded, $1<p<q<1+4 / N$, and $\varphi_{0} \in H$. Then there exists a unique solution $\varphi(t, x)$ of the Cauchy problem $(1.1),(2.1)$ in $([0, \infty) ; H)$, and $\varphi(t, \cdot)$ satisfies the following two conservation laws of the mass

$$
M(\varphi)=\int|\varphi|^{2} d x=\int\left|\varphi_{0}\right|^{2} d x=M\left(\varphi_{0}\right)
$$

and energy

$$
E(\varphi)=\int|\nabla \varphi|^{2}+V(x)|\varphi|^{2}-\frac{2 \mu}{p+1}|\varphi|^{p+1}-\frac{2 \lambda}{q+1}|\varphi|^{q+1} d x=E\left(\varphi_{0}\right)
$$

for all $t \in[0, \infty)$. 
Lemma 2.2. If $V(x) \rightarrow \infty$ as $|x| \rightarrow \infty$, let $1 \leq p<(N+2) /(N-2)$ when $N \geq 3$ and $1 \leq p<\infty$ when $N=1,2$. Then the embedding $H \hookrightarrow L^{p+1}\left(\mathbb{R}^{N}\right)$ is compact.

Proof. We firstly show it for $p=1$.

Since $H \hookrightarrow H^{1}\left(\mathbb{R}^{N}\right)$ continuously, it follows from the Sobolev embedding theorem that $H \hookrightarrow L^{p+1}\left(\mathbb{R}^{N}\right)$ continuously. Now let $\left\{u_{n}\right\}_{n} \subset H$ be a sequence such that

$$
u_{n} \rightarrow 0 \quad \text { weakly in } H \text {. }
$$

Then we have

$$
u_{n} \rightarrow 0 \quad \text { weakly in } H^{1}\left(\mathbb{R}^{N}\right) .
$$

Moreover, we have $M:=\sup _{n}\left\|u_{n}\right\|_{H}<\infty$. Let $\varepsilon>0$. Then there exists $B>0$ such that $1 / V(x) \leq \varepsilon$ for $|x| \geq B$. For $B$, from (2.7), we have

$$
u_{n} \longrightarrow 0 \quad \text { in } L^{2}(\{|x| \leq B\}) .
$$

It follows that there exists $m>0$ such that

$$
\int_{|x| \leq B}\left|u_{n}\right|^{2} d x \leq \varepsilon \quad \text { for } n \geq m .
$$

Then when $n \geq m$, we get

$$
\begin{aligned}
\int\left|u_{n}\right|^{2} d x & =\int_{|x| \leq B}\left|u_{n}\right|^{2} d x+\int_{|x| \geq B}\left|u_{n}\right|^{2} d x \\
& \leq \varepsilon+\varepsilon \int_{|x| \geq B} V(x)\left|u_{n}\right|^{2} d x \leq \varepsilon+\varepsilon C M^{2} .
\end{aligned}
$$

Here and hereafter $C$ denotes various positive constant. Thus we get that

$$
u_{n} \longrightarrow 0 \quad \text { in } L^{2}\left(\mathbb{R}^{N}\right) .
$$

It follows that the embedding $H \hookrightarrow L^{2}\left(\mathbb{R}^{N}\right)$ is compact.

For $p>1$, using the conclusion of $p=1$ and the Gagliardo-Nirenberg inequality,

$$
\|u\|_{L^{p+1}\left(\mathbb{R}^{N}\right)}^{p+1} \leq C\|\nabla u\|_{L^{2}\left(\mathbb{R}^{N}\right)}^{N(p-1) / 2}\|u\|_{L^{2}\left(\mathbb{R}^{N}\right)}^{p+1-N(p-1) / 2},
$$

we can get the conclusion immediately.

\section{The existence of standing waves}

Firstly, we define a variational problem as follows:

$$
d_{\rho}:=\inf _{\left\{u \in H \backslash\{0\}: \int|u|^{2} d x=\rho\right\}} E(u) \quad \text { for any } \rho>0 .
$$

Theorem 3.1. If $1<p<q<1+4 / N$, then

$$
d_{\rho}=\min _{\left\{u \in H \backslash\{0\}:\left\{|u|^{2} d x=\rho\right\}\right.} E(u) \text { for any } \rho>0 .
$$


Proof. Choose the minimizing sequence $\left\{u_{n}\right\}_{n \in \mathbb{N}}$ of the variational problem (3.1). Therefore, we have

$$
\begin{gathered}
u_{n} \in H \backslash\{0\}, \quad E\left(u_{n}\right) \longrightarrow d \quad \text { as } n \longrightarrow \infty, \\
\int\left|u_{n}\right|^{2} d x=\rho .
\end{gathered}
$$

By the Gagliardo-Nirenberg inequality and (3.4), for $1<p<q<1+4 / N$, one has

$$
\int\left|u_{n}\right|^{p+1} d x \leq C\left(\int\left|\nabla u_{n}\right|^{2} d x\right)^{\theta_{1}}, \quad \int\left|u_{n}\right|^{q+1} d x \leq C\left(\int\left|\nabla u_{n}\right|^{2} d x\right)^{\theta_{2}}
$$

where $0<\theta_{1}<\theta_{2}<1$. Hence, from (3.3) and (3.5), we have

$$
\begin{aligned}
C \geq & \int\left|\nabla u_{n}\right|^{2}+V(x)\left|u_{n}\right|^{2}-\frac{2 \mu}{p+1}\left|u_{n}\right|^{p+1}-\frac{2 \lambda}{q+1}\left|u_{n}\right|^{q+1} d x \\
\geq & \frac{1}{2} \int\left|\nabla u_{n}\right|^{2} d x-C\left(\int\left|\nabla u_{n}\right|^{2} d x\right)^{\theta_{1}}+\frac{1}{2} \int\left|\nabla u_{n}\right|^{2} d x-C\left(\int\left|\nabla u_{n}\right|^{2} d x\right)^{\theta_{2}} \\
& +\int(V(x)-\inf V(x))\left|u_{n}\right|^{2} d x+\int \inf V(x)\left|u_{n}\right|^{2} d x .
\end{aligned}
$$

Let $f(x)=x-C x^{\theta}$ and $x>0$, where $\theta \in(0,1)$ and $C>0$. One has

$\left(1^{0}\right)$ when $x=0$ or $x=C^{1 /(1-\theta)}, f(x)=0$;

$\left(2^{0}\right) f^{\prime}(x)=1-C \theta x^{\theta-1}$ and $f^{\prime}\left(C^{1 /(1-\theta)}\right)=1-\theta>0$;

$\left(3^{0}\right) f^{\prime \prime}(x)=C \theta(1-\theta) x^{\theta-2}>0$ as $x>0$.

From the Taylor expansion of $f(x)$,

$$
f(x)=f\left(x_{0}\right)+f^{\prime}\left(x_{0}\right)\left(x-x_{0}\right)+\frac{f^{\prime \prime}(\xi)}{2}\left(x-x_{0}\right)^{2},
$$

where $\xi$ is between $x_{0}$ and $x$, and choosing $x_{0}=C^{1 /(1-\theta)}$, one has

$$
f(x) \geq(1-\theta) x-(1-\theta) C^{1 /(1-\theta)} .
$$

Therefore, by (3.4), (3.6), and (3.8), it yields that $\left\{u_{n}\right\}_{n \in \mathbb{N}}$ is bounded in $H$. Therefore, there exists $u \in H$ such that the subsequence of $\left\{u_{n}\right\}_{n \in \mathbb{N}}$ which we still denote by $\left\{u_{n}\right\}_{n \in \mathbb{N}}$ satisfies

$$
u_{n} \rightarrow u \text { in } H
$$

By Lemma 2.2, one has

$$
\begin{gathered}
u_{n} \longrightarrow u \quad \text { in } L^{2}\left(\mathbb{R}^{N}\right), \\
u_{n} \longrightarrow u \quad \text { in } L^{p+1}\left(\mathbb{R}^{N}\right), \quad \text { in } L^{q+1}\left(\mathbb{R}^{N}\right) .
\end{gathered}
$$


Therefore, it follows from (3.4) and (3.10) that

$$
\int|u|^{2} d x=\rho
$$

which implies that $E(u) \geq d_{\rho}$. From (3.10) and with

$$
F(u):=\int|\nabla u|^{2}+(V(x)-\inf V(x))|u|^{2} d x
$$

being coercive and convex, one has

$$
F(u) \leq \lim _{n \rightarrow \infty} \inf F\left(u_{n}\right) .
$$

From (3.3), (3.9), (3.10), (3.11), and (3.13), it follows that $E(u)=d_{\rho}$. The proof is complete.

For any $\rho>0$, let $\Omega_{\rho}$ denote the set of the minimizers of the variational problem (3.2). Then for any $u \in \Omega_{\rho}$, by Theorem 3.1, there must exist a Lagrange multiplier $w$ such that

$$
-\triangle u+V(x) u+w u-\mu|u|^{p-1} u-\lambda|u|^{q-1} u=0 .
$$

It follows that $\varphi(t, x)=e^{i w t} u(x)$ is the standing wave solution of (1.1), which also called ground state since $u$ is a minimizer of (3.2). Thus $e^{i w t} u(x)$ is the orbit of $u$. It is obvious that for any $t \geq 0$, if $u$ is a solution of (3.2), then $e^{i w t} u$ is also a solution of (3.2), which yields $e^{i w t} u \in \Omega_{\rho}$.

\section{Orbital stability of standing waves}

Now in terms of Cazenave and Lion's argument [6], we have the following orbital stability.

Theorem 4.1. Assume that $V(x)$ satisfies that inf $V>-\infty, V(x) \rightarrow \infty$ as $|x| \rightarrow \infty$ and for each $|\alpha| \geq 2,\left|D^{\alpha} V\right|$ is bounded. Let $1<p<q<1+4 / N$. Then the standing waves of the Cauchy problem (1.1), (2.1) are orbitally stable. In other words, for arbitrary $\varepsilon>0$, there exists a $\sigma>0$ such that for any $\varphi_{0} \in H$, if

$$
\inf _{u \in \Omega_{\rho}}\left\|\varphi_{0}-u\right\|_{H}<\sigma
$$

then

$$
\inf _{u \in \Omega_{\rho}}\|\varphi(x, t)-u(x)\|_{H}<\varepsilon \quad \forall t \geq 0
$$

Proof. Firstly, for any $\varphi_{0} \in H$, from Lemma 2.1, the corresponding solution $\varphi(x, t)$ of the Cauchy problem (1.1), (2.1) is global and bounded in $H$. Now arguing by contradiction, if the conclusion of the theorem does not hold, then there exist a $\varepsilon_{0}>0$, a sequence $\left\{\varphi_{0}^{n}\right\}_{n \in \mathbb{N}} \subset H$ such that

$$
\inf _{u \in \Omega_{\rho}}\left\|\varphi_{0}^{n}-u\right\|_{H}<\frac{1}{n}
$$


and a sequence $\left\{t_{n}\right\}_{n \in \mathbb{N}}$ such that

$$
\inf _{u \in \Omega_{\rho}}\left\|\varphi_{n}\left(t_{n}, \cdot\right)-u(\cdot)\right\|_{H} \geq \varepsilon_{0}
$$

where $\varphi_{n}$ denotes the solution of the Cauchy problem (1.1), (2.1) with the initial value $\varphi_{0}^{n}$.

From (4.3) and Lemma 2.2, we have

$$
\begin{gathered}
M\left(\varphi_{0}^{n}\right)=\int\left|\varphi_{0}^{n}\right|^{2} d x \longrightarrow \int|u|^{2} d x, \\
E\left(\varphi_{0}^{n}\right) \longrightarrow E(u) .
\end{gathered}
$$

It follows from (4.5) and the conservation laws in Lemma 2.1 that $\left\{\varphi_{n}(t, \cdot)\right\}_{n \in \mathbb{N}}$ is a minimizing sequence for the problem (3.2). Therefore, there exists a $u \in \Omega_{\rho}$ such that

$$
\left\|\varphi_{n}\left(t_{n}, \cdot\right)-u\right\|_{H} \longrightarrow 0 \quad \text { as } n \longrightarrow \infty
$$

This is contradictory with (4.4). The proof is complete.

\section{Acknowledgment}

This work is supported by National Natural Science Foundation (10271084), SZD0406, and the Emphasis Scientific Research Foundation of Sichuan Province.

\section{References}

[1] H. Berestycki and T. Cazenave, Instabilité des états stationnaires dans les équations de Schrödinger et de Klein-Gordon non linéaires, Comptes Rendus des Séances de l'Académie des Sciences. Série I. Mathématique 293 (1981), no. 9, 489-492 (French).

[2] C. C. Bradley, C. A. Sackett, and R. G. Hulet, Bose-Einstein condensation of Lithium: observation of limited condensate number, Physical Review Letters 78 (1997), 985-989.

[3] R. Carles, Critical nonlinear Schrödinger equations with and without harmonic potential, Mathematical Models \& Methods in Applied Sciences 12 (2002), no. 10, 1513-1523.

[4] _ Remarks on nonlinear Schrödinger equations with harmonic potential, Annales Henri Poincaré. A Journal of Theoretical and Mathematical Physics 3 (2002), no. 4, 757-772.

[5] T. Cazenave, An Introduction to Nonlinear Schrödinger Equations, Textos de Metodos Matematicos, vol. 26, IMUFRJ, Rio de Janeiro, 1996.

[6] T. Cazenave and P.-L. Lions, Orbital stability of standing waves for some nonlinear Schrödinger equations, Communications in Mathematical Physics 85 (1982), no. 4, 549-561.

[7] F. Dalfovo, S. Giorgini, L. P. Pitaevskii, and S. Stringari, Theory of Bose-Einstein condensation in trapped gases, Reviews of Modern Physics 71 (1999), no. 3, 463-512.

[8] W. Y. Ding and W.-M. Ni, On the existence of positive entire solutions of a semilinear elliptic equation, Archive for Rational Mechanics and Analysis 91 (1986), no. 4, 283-308.

[9] D. Fujiwara, Remarks on convergence of the Feynman path integrals, Duke Mathematical Journal 47 (1980), no. 3, 559-600.

[10] M. Hirose and M. Ohta, Structure of positive radial solutions to scalar field equations with harmonic potential, Journal of Differential Equations 178 (2002), no. 2, 519-540.

[11] Y. Kagan, A. E. Muryshev, and G. V. Shlyapnikov, Collapse and Bose-Einstein condensation in a trapped Bose gas with negative scattering length, Physical Review Letters 81 (1998), 933-937. 
[12] H. A. Levine, Instability and nonexistence of global solutions to nonlinear wave equations of the form $P u_{t t}=-A u+\mathscr{F}(u)$, Transactions of the American Mathematical Society 192 (1974), 121.

[13] Y.-G. Oh, Cauchy problem and Ehrenfest's law of nonlinear Schrödinger equations with potentials, Journal of Differential Equations 81 (1989), no. 2, 255-274.

[14] L. E. Payne and D. H. Sattinger, Saddle points and instability of nonlinear hyperbolic equations, Israel Journal of Mathematics 22 (1975), no. 3-4, 273-303.

[15] P. H. Rabinowitz, On a class of nonlinear Schrödinger equations, Zeitschrift für Angewandte Mathematik und Physik. ZAMP 43 (1992), no. 2, 270-291.

[16] W. A. Strauss, Nonlinear Wave Equations, CBMS Regional Conference Series in Mathematics, vol. 73, American Mathematical Society, Rhode Island, 1989.

[17] T. Tsurumi and M. Wadati, Collapses of wave functions in multidimensional nonlinear Schrödinger equations under harmonic potential, Journal of the Physical Society of Japan 66 (1997), no. 10, 3031-3034.

[18] M. I. Weinstein, Nonlinear Schrödinger equations and sharp interpolation estimates, Communications in Mathematical Physics 87 (1982/1983), no. 4, 567-576.

[19] K. Yajima, On fundamental solution of time dependent Schrödinger equations, Advances in Differential Equations and Mathematical Physics (Atlanta, GA, 1997), Contemp. Math., vol. 217, American Mathematical Society, Rhode Island, 1998, pp. 49-68.

[20] J. Zhang, Stability of attractive Bose-Einstein condensates, Journal of Statistical Physics 101 (2000), no. 3-4, 731-746.

[21]__ Stability of standing waves for nonlinear Schrödinger equations with unbounded potentials, Zeitschrift für Angewandte Mathematik und Physik. ZAMP 51 (2000), no. 3, 498-503.

[22] _ Cross-constrained variational problem and nonlinear Schrödinger equation, Foundations of Computational Mathematics-Proceedings of the Smalefest (Hong Kong, 2000), World Scientific Publishing, New Jersey, 2002, pp. 457-469.

[23] _ On the standing wave in coupled non-linear Klein-Gordon equations, Mathematical Methods in the Applied Sciences 26 (2003), no. 1, 11-25.

Guanggan Chen: College of Mathematics and Software Science, Sichuan Normal University,

Chengdu 610066, China

E-mail address: chenguanggan@hotmail.com

Jian Zhang: College of Mathematics and Software Science, Sichuan Normal University, Chengdu 610066, China

E-mail address: jianzhan@mail.sc.cninfo.net

Yunyun Wei: College of Information Management, Chengdu University of Technology,

Chengdu 610059, China

E-mail address: yunyunwei@sina.com 


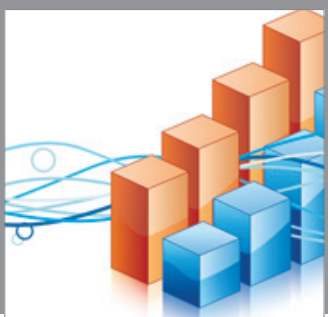

Advances in

Operations Research

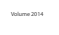

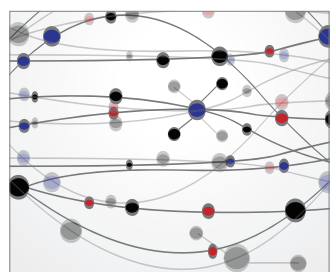

\section{The Scientific} World Journal
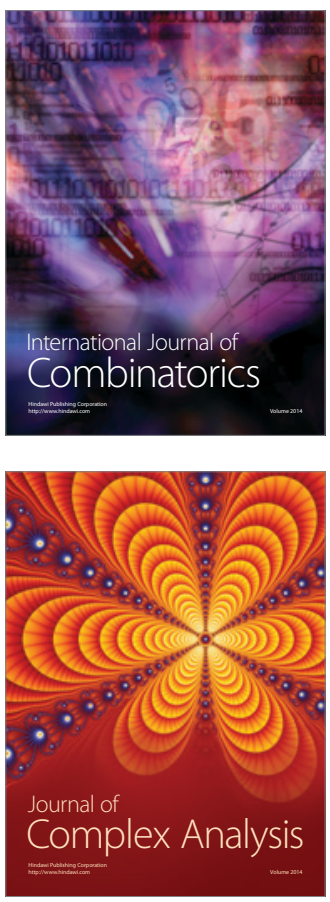

International Journal of

Mathematics and

Mathematical

Sciences
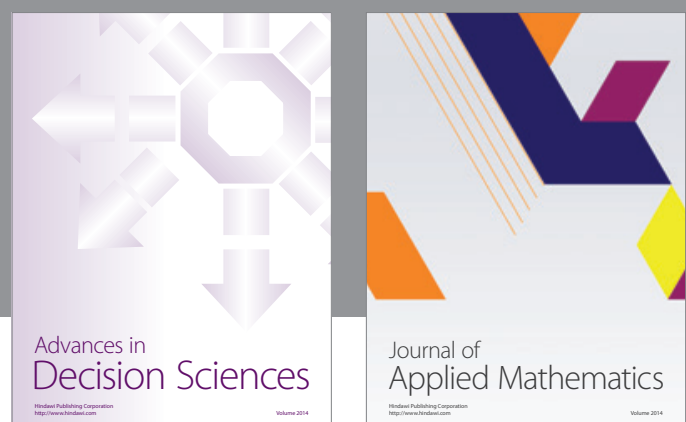

Journal of

Applied Mathematics
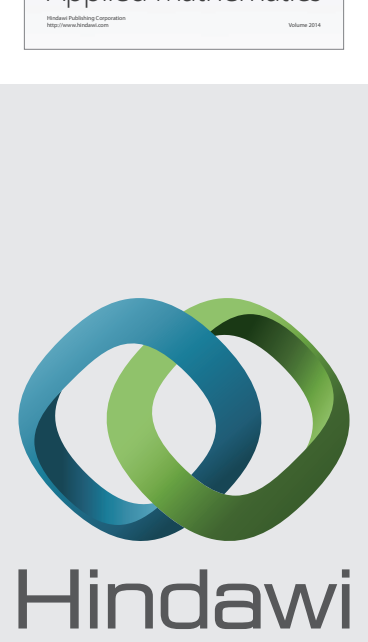

Submit your manuscripts at http://www.hindawi.com
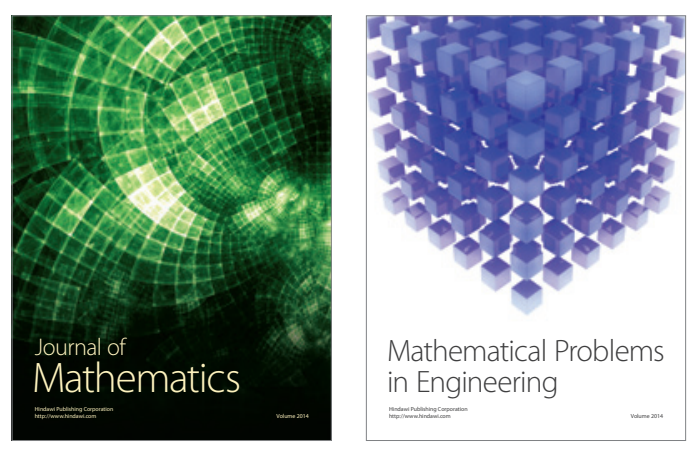

Mathematical Problems in Engineering
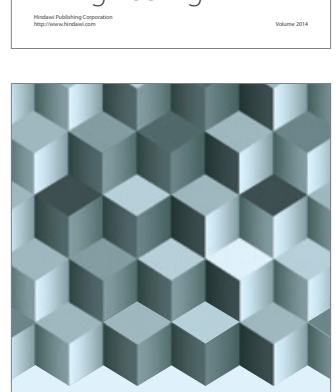

Journal of

Function Spaces
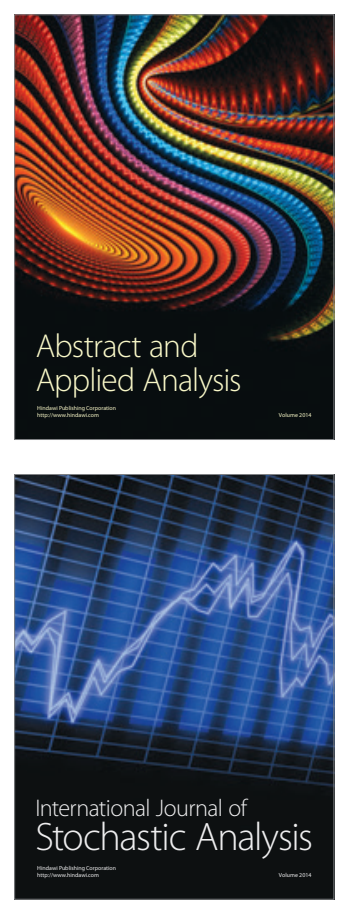

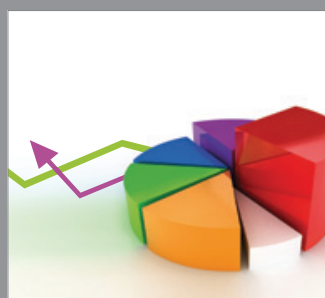

ournal of

Probability and Statistics

Promensencen
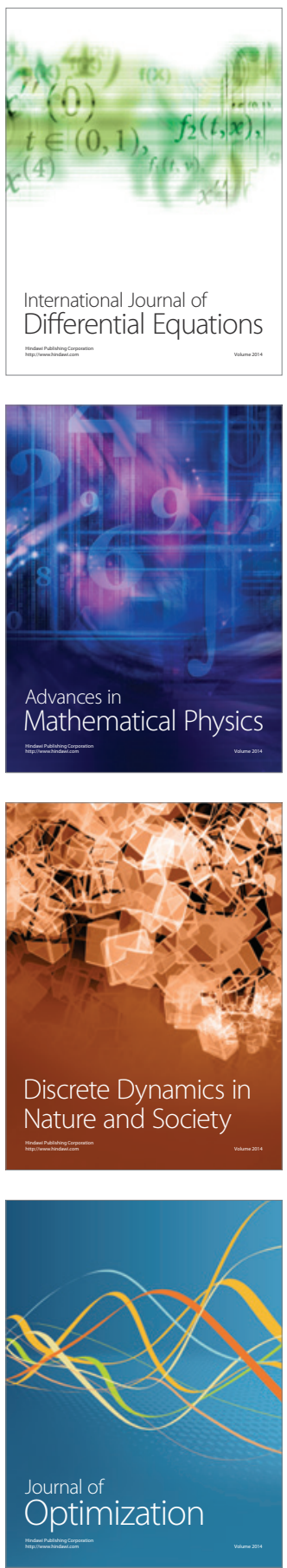